\title{
Crecimiento y topología de la ramificación de la guanábana y el manirote
}

\author{
Jorge Parés-Martínez ${ }^{(1)}$, Miguel Arizaleta(1) y Damaso Bautista(1) $^{(1)}$
}

\begin{abstract}
(1)Universidad Centroccidental "Lisandro Alvarado", Decanato de Agronomía, Dpto. Fitotecnia, Estado Lara, Venezuela. E-mail: jorgepares@ucla.edu.ve, miguelarizaleta@ucla.edu.ve
\end{abstract}

\begin{abstract}
Resumen - El objetivo de este trabajo fue caracterizar el crecimiento y el sistema de ramificación de la parte aérea de la guanábana (Annona muricata) y el manirote (Annona montana) a pie franco y Annona muricata injertada sobre Annona montana. Se evaluó la longitud, el número de nudos y el número de brotes producidos por ramas de primer y segundo orden, después de haberlas sometido a poda. También se realizó un análisis topológico de la ramificación, a los cuatro meses después de la primera y segunda poda, mediante la determinación de las características altitud, magnitud y total de ramificaciones externas, para establecer la configuración de la copa. Las ramas de los materiales evaluados presentaron tendencias similares en cuanto a la longitud total y la formación de nudos, además de ritmicidad en el alargamiento. La formación de brotes aumentó gradualmente con el tiempo y la producción de ramas potencialmente productivas de la A. muricata a pie franco fue mayor que en las plantas injertadas. El material vegetal evaluado mostró un patrón de ramificación dicotómico y numéricamente escaso. Las características topológicas utilizadas definieron satisfactoriamente el tipo de ramificación que tomaron las plantas, durante la primera y segunda poda.
\end{abstract}

Términos para indexación: Anonna muricata, Anonna montana, características topológicas, poda.

\section{Growth and topological branching of the soursop and manirote}

\begin{abstract}
The objective of this work was to characterize growth and branching system of the soursop (Annona muricata) and manirote (Annona montana) rootstock plants, and grafted plants of Annona muricata on Annona montana. Length and number of branchs and node number produced by branches of first and second order, after each of two sequential pruning, were determined. A topologycal analysis of branching was performed after the first and second pruning, in which altitude, magnitude and total number of external branching were measured. Branches of the three evaluated materials presented similar tendency related to the length, node formation and lengthening. The formation of node increased gradually in time and the production of potentially productive branches of the $A$. muricata rootstock plants was greater than grafted ones. Plants showed a pattern of dichotomic and numerically scarce branching. The topologycal characteristics defined satisfactorily the type of branching that rootstock plants presented during the first and second pruning.
\end{abstract}

Index terms: Annona muricata, Annona montana, topological characteristic, pruning.

\section{Introducción}

Los estudios sobre la dinámica del crecimiento y arquitectura de árboles tropicales fueron iniciados por Hallé y Oldeman en 1970, quienes consideraron a las ramas como las unidades básicas de la arquitectura de los árboles (Hallè et al., 1978). Avilán et al. (1995) señalaron que es necesario conocer detalladamente la forma de crecimiento de la copa de las plantas de tipo arbóreo y los tipos de ramas que conforman su estructura, para establecer prácticas culturales relacionadas con la conducción y poda. El crecimiento de un vegetal puede ser rítmico o continuo. El rítmico ocurre cuando el eje presenta periodicidad de elongación, mientras que el continuo ocurre cuando el eje no presenta periodicidad de alargamiento (Caraglio \& Barthélémy, 1997). La guanábana exhibe entre uno y tres flujos de crecimiento al año, presentando éstos estrecha relación con la distribución de la precipitación (Escobar \& Sánchez, 1992; Yamarte, 2001).

Las ramas se consideran, en sentido topológico, de un orden mayor al del eje en el cual se insertan. Corrientemente, se considera al eje principal como el punto de partida, de orden cero, de allí en adelante se 
les designa con la numeración ordinaria (Lindorf et al., 1991). La ramificación puede ser básicamente de dos tipos: dicótoma y lateral. En la primera, el centro del ápice vegetativo se divide en dos nuevos puntos meristemáticos, mientras que la ramificación lateral se origina por la actividad de los meristemos laterales, dando como consecuencia la ramificación monopódica, donde el eje principal domina sobre las ramas laterales y la ramificación simpódica, donde las ramas laterales se desarrollan más que el eje principal, ya sea por sustitución o por aposición de la yema terminal (Lindorf et al., 1991).

Fitter (1987) y Martín et al. (1995) señalaron que la copa de la planta esta constituida por eslabones, los cuales son porciones lineales de brotes entre un meristema y el punto de ramificación más cercano, dos puntos de ramificación proximales, o un punto de ramificación y el tronco de la planta. Los mismos autores mencionaron que los eslabones pueden ser externos, si finalizan en un meristemo, o internos si finalizan en otro eslabón.

En las plantas, los eslabones se encuentran distribuidos en patrones definidos, conformando la estructura de la red de ramas que configuran la parte aérea dentro de una topología particular (Fitter et al., 1991), la cual puede ser definida mediante la determinación de las características topológicas: altitud, magnitud y total de ramificaciones externas, establecidas por Fitter (1987). La altitud se refiere al número que le corresponde al eslabón más distante, asignados a través del contaje de los eslabones internos, desde la base de la planta. La magnitud se define como la sumatoria de todos los eslabones externos, mientras que el total de ramificaciones externas es la sumatoria de los números que les corresponden a los eslabones externos, en todos los posibles recorridos.

Según Fitter (1987) y Fitter et al. (1991), para un mismo conjunto de ramificaciones varias topologías pueden ser posibles, las cuales conforman grupos topológicamente distintos de acuerdo a los valores de las características altitud, magnitud y total de ramificaciones externas, que varían entre límites. El límite mínimo está asociado a un patrón de ramificación escaso al eje principal y consistente de ramificación simpódica, definida esta topología como dicotómica, mientras que su máximo está asociado con un patrón de ramificación profuso al eje principal y consistente de ramificación monopódica. Esta topología ha sido definida como espina de pescado. Patrones de ramificación intermedios a los valores teóricos extremos han sido definidos como aleatorios.
El objetivo del presente trabajo fue evaluar el crecimiento de las ramas y establecer el patrón topológico de ramificación, posterior a las podas, de la A. muricata y A. montana, a pie franco y de la A. muricata injertada sobre A. montana.

\section{Material y Metodos}

Esta investigación fue conducida en la Estación Experimental Miguel Luna Lugo, Universidad Centroccidental "Lisandro Alvarado", Municipio Palavecino, Estado Lara, $\left(10^{\circ} 1{ }^{\prime} 25^{\prime \prime} \mathrm{N}\right.$; 69 ${ }^{\circ} 17^{\prime} \mathrm{O}$; $510 \mathrm{msnm})$. El régimen pluviométrico promedio es de aproximadamente $870 \mathrm{~mm} / \mathrm{año}$; con temperatura promedio anual de $25,2^{\circ} \mathrm{C}$ y la evaporación dos veces superior a la precipitación.

Se evaluaron plantas de A. muricata y A. montana a pie franco y A. muricata injertada sobre $A$. montana. Estas fueron sometidas a una primera poda de formación, la cual consistió en el corte del eje principal a una altura de $60 \mathrm{~cm}$ desde suelo, con la eliminación de las ramas de las plantas, a excepción de 3 a 4 ramas ubicadas entre los 30 y $60 \mathrm{~cm}$ desde el suelo (Sacramento \& Vieira, 1997). Esta poda se realizó cuando las plantas tenían una altura aproximada de 1 metro. Posteriormente, se realizó la segunda poda, a los cuatro meses de la primera, mediante el despuntado de las ramas de primer orden. Así, cada planta quedó conformada por un eje principal de orden cero, ramas laterales de primer orden después de la primera poda y ramas laterales de segundo orden, después de la segunda poda.

El ensayo se estableció en un diseño de bloques al azar, con tres tratamientos, 5 repeticiones y dos plantas por unidad experimental; se consideraron como tratamientos a los materiales vegetales evaluados. Las labores agronómicas en el ensayo se realizaron de manera similar a las habituales en el cultivo.

En el estudio se determinó el crecimiento longitudinal, así como también el número de nudos y de brotes, formados tanto por las ramas de primer, como por las de segundo orden. Estas variables se evaluaron quincenalmente, durante cuatro meses después de cada poda. Se estudiaron, por planta, 4 ramas de primer orden después de la primera poda y 2 ramas de segundo orden después de la segunda poda.

Para establecer el patrón de ramificación, se procedió a calcular los valores máximos y mínimos de las características topológicas: altitud, magnitud y total de ramificaciones externas, de acuerdo a las ecuaciones 
establecidas por Fitter (1996). La altitud máxima es igual a la magnitud y la mínima es igual a $(\log 2(\mu-1))+2$. El valor máximo del total de ramificaciones externas es igual a $0,5\left(\mu^{2}+3 \mu-2\right)$, mientras que el mínimo es igual a $\mu(\min (a)+1)-2^{\min (a)-1}$.

La determinación de las características topológicas altitud, magnitud y total de ramificaciones externas se realizaron a los cuatro meses, después de la primera y de la segunda poda, siguiéndose la metodología descrita por Fitter (1987).

Los datos correspondientes a la característica topológica magnitud fueron sometidos al análisis de varianza propio del diseño de bloques al azar, previa comparación del cumplimiento de los supuestos del análisis. Cuando se determinaron diferencias estadísticas entre los tratamientos, se realizaron pruebas de comparaciones múltiples de medias, de acuerdo a Duncan $(\mathrm{P} \leq 0,05)$. Fueron utilizados para estos análisis los paquetes estadísticos Statistix V 4.04 y SAS, V 6.12. En el caso de las variables longitud total, número de nudos y brotes producidos por los ejes evaluados, se analizaron los gráficos a fin de describir su comportamiento a través del tiempo.

\section{Resultados y Discusión}

Las plantas de A. muricata a pie franco presentaron las mayores tasas de crecimiento longitudinal de las ramas de primer orden, durante todo el período de evaluación; mientras que las ramas del resto de las plantas estudiadas presentaron tasas de crecimiento menores y similares entre sí, hasta la décima semana, momento a partir del cual las injertadas mostraron un incremento en la tasa de crecimiento (Figura 1). Aparentemente, después de la primera poda, el crecimiento formó una curva bisigmoidal en los materiales considerados, con una primera fase de crecimiento rápido que transcurrió entre la poda y la sexta semana, una fase de crecimiento lento entre la sexta y la doceava semana, seguida de una nueva fase de crecimiento rápido de la semana doce a la dieciséis. Este último flujo de crecimiento pudo ser inducido por lluvias. Es conocido que los flujos de crecimiento de la guanábana se correlacionan estrechamente con las precipitaciones (Escobar \& Sánchez, 1992; Yamarte, 2001).

El crecimiento de las ramas de segundo orden de las plantas a pie franco presentó un comportamiento muy similar entre si, siendo mayor al de las ramas de las plantas injertadas (Figura 1). Después de la segunda poda, se observó un crecimiento muy lento durante las primeras cinco semanas, incrementándose a partir de la sexta semana. Fue notorio que durante los dos ciclos analizados, las ramas de primer y segundo orden presentaron periodicidad en su alargamiento. Tendencia similar fue observada por Yamarte (2001), al evaluar el crecimiento de ramas de plantas de A. muricata a pie franco.

Otro aspecto importante de observar es que el crecimiento de las ramas de las plantas injertadas fue menor, en comparación a la longitud de las ramas de A. muricata a pie franco, respuesta que podría ser consecuencia del tipo de propagación utilizada, ya que se señala a la injertación como una técnica para controlar el tamaño de las plantas de tipo arbóreo (Avilán et al., 1997).

El número de nudos formados presentó tendencia similar al crecimiento longitudinal de las ramas. La formación de nudos de las ramas de primer orden se detuvo entre la sexta y octava semana, para la A. muricata a pie franco, y entre la octava y décima semana para el resto de los materiales (Figura 1). Las ramas de origen secundario de las plantas a pie franco presentaron mínima formación de nudos durante las cuatro primeras semanas, después de la segunda poda; las ramas de las plantas injertadas iniciaron la producción de nudos después de la sexta semana de realizada la poda (Figura 1). Yamarte (2001) reportó que la disminución en la cantidad de nudos, producidos por las ramas de las plantas de $A$. muricata, es debida a las condiciones agroclimáticas, específicamente a limitaciones en la disponibilidad de humedad del suelo, como consecuencia de la disminución de la precipitación.

La formación de brotes aumentó gradualmente en el tiempo, después de efectuadas las podas en cada uno de los materiales evaluados (Figura 1). Sin embargo, la tendencia a la formación de brotes en las ramas secundarias fue de menor intensidad, en comparación a las de primer orden. Adicionalmente, la tasa de formación de ramas laterales de las plantas a pie franco fue mayor a la presentada por las injertadas. Estos resultados podrían indicar que la producción de ramas, potencialmente productivas en las plantas de A. muricata a pie franco, tiende a ser mayor que en las plantas injertadas. La A. montana aunque inició, en las ramas de segundo orden, con un número de brotes laterales similar que las plantas injertadas, incrementó rápidamente el número de sus brotes superando, a partir de la décima semana, la producción de brotes de la $A$. muricata, lo que indica 

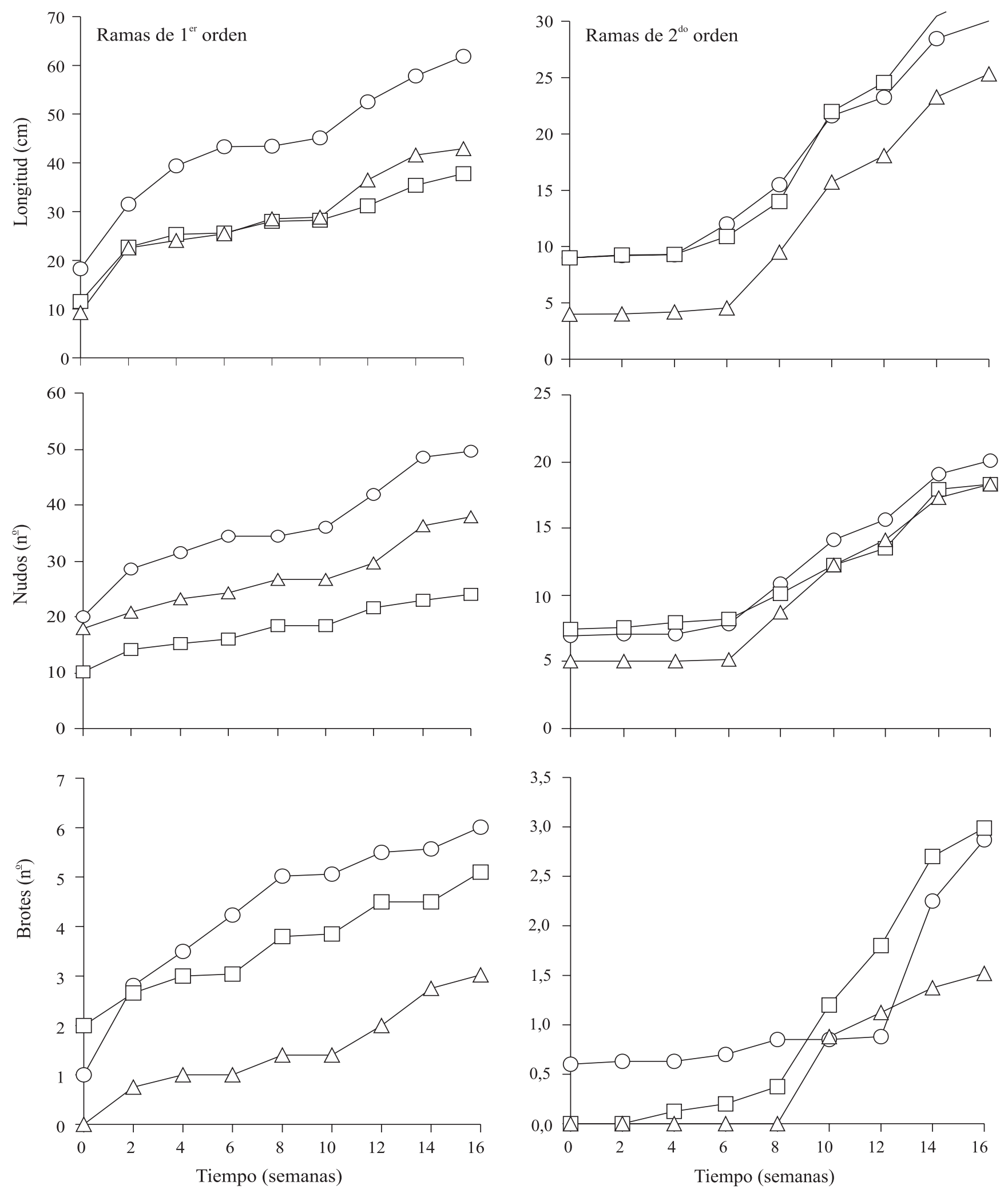

Figura 1. Crecimiento longitudinal, número de nudos y número de brotes de las ramas de $1^{\text {er }}$ y $2^{\text {do }}$ orden, durante 4 meses a partir de la primera $(n=40)$ y segunda poda $(n=20)$, en plantas de Annona muricata $(O), A$. montana $(\square)$ y A. muricata/A. montana $(\triangle)$. 
que la A. montana tiene mayor capacidad de ramificación que la $A$. muricata a pie franco o injertada sobre la $A$. montana (Figura 1 ).

Se podría señalar que al aumentar el número de puntos potenciales de brotación, después de cada poda, la competencia por asimilados se hace crítica, lo que limita la capacidad de brotación (Coutin \& Razeto, 1999). Las interrelaciones injerto-patrón imponen ciertas limitaciones a la producción de ramas, por el control que se ejerce sobre el crecimiento. Además, la diferencia en la producción de las ramas laterales puede depender, en algunos casos, de la competencia por fotoasimilados, elementos nutritivos, estrés hídrico, capacidad reproductiva de las yemas y/o condiciones agroclimáticas, entre otras (Coutin \& Razeto, 1999; Yamarte, 2001).

En el Cuadro 1 están representados los valores de las características topológicas para el material vegetal evaluado cuatro meses después de la primera poda. Como se observa, la magnitud $(\mu)$ presentó diferencias significativas y al ser sometida a la prueba de medias se establecieron dos grupos topológicos, el primero formado por las plantas a pie franco y el segundo por las injertadas.

Según los criterios establecidos por Fitter et al. (1991), se determinó que el material evaluado presentó ramificación dicótoma, del tipo escamosa y simpódica, por cuanto los valores medios de las características altitud y total de ramas externas tendieron a los valores mínimos. A pesar de que estadísticamente se obtuvieron dos grupos, ambos con similar patrón de ramificación, es necesario destacar que el grupo topológico (A), conformado por las plantas a pie franco, presentó mayor número de eslabones en su máxima trayectoria de ramificación en comparación a las plantas injertadas. Esta tendencia de ramificación fue aún mayor en las ramas de las plantas a pie franco, dado que el total de ramificaciones externas superó ampliamente el valor del grupo B. Los resultados corresponden a la ramificación

Cuadro 1. Valores medios, máximos y mínimos de la altitud (a) y del total de ramificaciones externas $(\mathrm{Pe})$ de la primera poda, en cada uno de los materiales vegetales evaluados ${ }^{(1)}$.

\begin{tabular}{lrrrrrrrr}
\hline \multirow{2}{*}{ Material vegetal } & \multicolumn{2}{c}{ Medios } & & \multicolumn{2}{c}{ Máximos $(\mu)$} & & \multicolumn{2}{c}{ Mínimos } \\
\cline { 2 - 3 } & \multicolumn{1}{c}{$\mathrm{a}$} & $\mathrm{Pe}$ & $\mathrm{a}$ & $\mathrm{Pe}$ & $\mathrm{a}$ & $\mathrm{Pe}$ \\
\hline Annona muricata pie franco & 11,8 & 176,9 & & $23,0 \mathrm{a}$ & 321,2 & & 3,6 & 98,4 \\
Annona montana pie franco & 10,6 & 165,2 & & $18,0 \mathrm{a}$ & 323,3 & & 3,6 & 96,9 \\
A. muricata/A. montana & 8,1 & 78,1 & & $13,3 \mathrm{~b}$ & 112,1 & & 3,4 & 53,6 \\
\hline
\end{tabular}

(1)Medias seguidas por las mismas letras no son significativamente diferentes entre sí, según la prueba de Duncan al 5\% de probabilidad. conformada después de la primera poda. En este caso, la mayoría de las ramas formadas corresponden a eslabones externos, lo que es una característica estimulada por la poda. Resultados similares reportaron Guerra \& Bautista (1999), al realizar la descripción topológica de la ramificación de clones de guayabo sometidos a poda.

En las plantas injertadas, la dominancia apical y algunos efectos originados de las interrelaciones patróninjerto parecen haber ejercido un efecto acentuado sobre la ramificación de las ramas de primer orden, causando diferencias entre la topología de las plantas injertadas, con respecto a la topología de las plantas a pie franco.

En el Cuadro 2, están representados los valores de las características topológicas, para el material vegetal evaluado cuatro meses después de la segunda poda. Se observa que la magnitud $(\mu)$ presentó diferencias significativas, estableciéndose tres grupos topológicos, el primero formado por la A. muricata a pie franco, el segundo por las plantas injertadas y el tercero por la A. montana.

Es necesario destacar que el grupo topológico (A) tuvo mayor número de eslabones en su máxima trayectoria de ramificación, en comparación al grupo topológico conformado por las plantas injertadas. Adicionalmente, esta tendencia de ramificación fue aún mayor en las ramas de las plantas a pie franco, ya que el valor total de ramificaciones externas de las plantas a pie franco superó el valor del grupo topológico de las plantas injertadas.

Al igual que en el caso anterior, las plantas evaluadas mantuvieron fielmente el tipo de ramificación dicótoma, caracterizada por un escaso número de ramas ya obtenido por efecto de la primera poda, por cuanto los valores medios de las características altitud y total de ramificaciones externas tendieron a acercarse a los valores mínimos. La ramificación dicotómica ocurrió por efecto de la segunda poda sobre la formación de las

Cuadro 2. Valores medios, máximos y mínimos de la altitud (a) y del total de ramificaciones externas (Pe) luego de la segunda $\operatorname{poda}^{(1)}$

\begin{tabular}{|c|c|c|c|c|c|c|}
\hline \multirow[t]{2}{*}{ Material vegetal } & \multicolumn{2}{|c|}{ Medios } & \multicolumn{2}{|c|}{ Máximos $(\mu)$} & \multicolumn{2}{|c|}{ Mínimos } \\
\hline & $\mathrm{a}$ & $\mathrm{Pe}$ & $\mathrm{a}$ & $\mathrm{Pe}$ & $\mathrm{a}$ & $\mathrm{Pe}$ \\
\hline Annona muricata pie franco & 28,0 & 2062,4 & $149,3 \mathrm{a}$ & 11802,7 & 4,4 & 759,2 \\
\hline Annona montana pie franco & 16,0 & 575,2 & $58,8 \mathrm{c}$ & 1878,0 & 4,1 & 289,9 \\
\hline A. muricata/A. montana & 22,7 & 815,4 & $72,8 \mathrm{~b}$ & 2776,0 & 4,1 & 366,7 \\
\hline
\end{tabular}

(1)Medias seguidas por las mismas letras no son significativamente diferentes entre sí, según la prueba de Duncan al 5\% de probabilidad. 
ramas de mayor orden, lo que indujo la brotación de las yemas, principalmente en los eslabones más externos de la copa. Además, se señala que la conducción aplicada a la copa estimula patrones de ramificación que no podrán ser aleatorios ni en espina de pez, debido a que las ramificaciones de los eslabones internos no participan en la formación de la copa (Guerra \& Bautista, 1999).

\section{Conclusiones}

1. La practica de la injertación disminuye el crecimiento longitudinal de las ramas de primer y segundo orden en plantas de A. muricata, así como en número de brotes producidos.

2. El crecimiento expresado en número de nudos presenta tendencia similar a la del crecimiento longitudinal de las ramas para cada uno de los materiales evaluados.

3. El material vegetal evaluado muestra un patrón de ramificación dicotómico, con escaso número de ramas, como consecuencia de la realización de las podas.

4. Las características topológicas utilizadas definen satisfactoriamente el tipo de ramificación que tomaron las plantas, durante la primera y segunda poda.

\section{Referencias}

AVILÁN, L.; LEAL, F.; BAUTISTA, D. El aguacatero: principios y técnicas para su producción. Caracas, Venezuela: Espasande, 1995. 380p.

AVILÁN, L.; RODRÍGUEZ, M.; RUÍZ, J.; MARÍN, C. Selección de patrones de bajo porte en mango. Agronomía Tropical, v.47, p.259-270, 1997.

CARAGLIO, Y.; BARTHÉLÉMY, D. Revue critique des termes relatifs à la croissance et à la ramification des tiges des végétaux vasculaires. In: BOUCHON, J.; RAFFYE, P.H.; BARTHÉLÉMY, D. (Ed.). Modélisation et simulation de l'architecture des végétaux. Paris: INRA, 1997. p.11-87.
COUTIN, R.; RAZETO, B. Evaluación del comportamiento de yemas de chirimoyo sometidas a tratamientos de floración forzada. In: CONGRESO INTERNACIONAL DE ANONÁCEAS, 2., 1999, México. Memorias. Chapingo, Mexico: Universidad Autónoma de Chapingo, 1999. p.58-72.

ESCOBAR, T.; SÁNCHEZ, L. Guanábano. In: GALLEGO, R. (Ed.). Fruticultura colombiana. Palmira, Colombia: Produmedios, 1992. p.225-230. (Manual de asistencia técnica, 57).

FITTER, A.H. An architectural approach to the comparative ecology of plant root systems. New Phytologist, v.106, p.61-77, 1987.

FITTER, A.H. Characteristics and function of root systems. In: WAISEL, Y.; ESHEL, A.; KAFKAFI, U. (Ed.). Plant roots: the hidden half. New York: Marcel Dekker, 1996. p.1-20.

FITTER, A.H.; STICKLAND, T.R.; HARVEY, M.L.; WILSON, G.W. Architectural analysis of plant root systems. 1. Architectural correlates of exploitation efficiency. New Phytologist, v.118, p.375382, 1991.

GUERRA, E.; BAUTISTA, D. Descripción topológica de la ramificación de tres clones de guayabo sometidos a poda. Agronomía Tropical, v.49, p.155-168, 1999.

HALLÈ, F.; OLDEMAN, R.A.A.; TOMLINSON, P.B. Tropical trees and forest: an architectural analysis. Berlin: Springer-Verlag, 1978. 496p.

LINDORF, H.; PARISCA, L.; RODRÍGUEZ, P. Botánica: clasificación, estructura y reproducción. Caracas, Venezuela: Universidad Central de Venezuela, 1991. 296p.

MARTÍN, C.; STUTZ, J.; KIMBALL, B.; IDSO, S.; AKEY, D. Growth and topological changes of Citrus limon (L.) Burm. F "Eureka" in response to high temperatures and elevated atmospheric carbon dioxide. Journal of the American Society for Horticultural Science, v.120, p.1025-1031, 1995.

SACRAMENTO, C.K.; VIEIRA, J.B. Formação do pomar e tratos culturais da graviola. In: REBOUÇAS, A.; SOUZA, I.V.; MAGALHÃES, O.; HOJO, T.N. (Ed.). Anonáceas: produção e mercado (pinha, graviola, atemóia e cherimóia). Vitória da Conquista, Bahia: UESB/DFZ, 1997. p.88-93.

YAMARTE, M. Estudio de crecimiento y fenología del guanábano (Annona muricata L.) bajo las condiciones de un bosque muy seco tropical. 2001. 71p. Disertación (Magíster) - La Universidad del Zulia, Maracaibo, Venezuela.

$\overline{\text { Recibido el } 20 \text { de mayo de } 2004 \text { y aceptado el } 19 \text { de julio de } 2005}$ 